SU-ITP-99/25

hep-th/9905210

May 1999

\title{
Bent Domain Walls as Braneworlds
}

\author{
Nemanja Kaloper円 \\ Department of Physics \\ Stanford University \\ Stanford, CA 94305-4060
}

\begin{abstract}
We consider domain walls embedded in curved backgrounds as an approximation for braneworld scenarios. We give a large class of new exact solutions, exhausting the possibilities for describing one and two walls for the cases where the curvature of both the bulk and the wall is locally constant. In the case of two walls, we find solutions where each wall has positive tension. An interesting property of these solutions is that the curvature of the walls can be much smaller than the tension, leading to a significant cancellation of the effective cosmological constant, which however is still much larger than the observational limits. We further discuss some aspects of inflation in models based on wall solutions.
\end{abstract}

\footnotetext{
1 email: kaloper@epic.stanford.edu
} 


\section{Introduction}

There has been considerable interest recently in higher dimensional theories with internal dimensions which are large compared to the fundamental Planck length of the theory [1][12]. If the fundamental theory is higher-dimensional, the higher-dimensional fundamental Planck scale may be many orders of magnitude smaller than the Planck scale in a world of only four dimensions $(4 D)$. The effective $4 D$ Planck mass is given by $M_{4}^{2} \sim\left(\frac{r_{0}}{l_{D}+4}\right)^{D} M_{D+4}^{2}$ where $r_{0}$ is the size of the compact dimensions [1]. If these dimensions are much larger than the fundamental Planck length, $\frac{r_{0}}{l_{D+4}}>>1$, the reduced Planck mass may be exponentially larger than the fundamental Planck mass. Setting the fundamental Planck mass to be equal to the unification scale of the theory, one may then understand the mass hierarchy as a consequence of stabilizing the internal space at a radius which is exponentially large compared to the fundamental scale. This scale has been elucidated in [2] as emerging from supersymmetry breaking problem in superstring theory.

In most of the models considered to date, the $4 D$ world is a domain wall, or 3-brane, living in a higher-dimensional theory with compact internal dimensions. The metric was independent of the internal coordinates. Similar proposals have been made before [13, 14], but recent developments in string and $M$-theory, which may explain why matter degrees of freedom are stuck to the wall [7, \&] gave new credibility to such ideas.

It has been pointed recently, however, that a different realization of the higherdimensional resolution of mass hierarchy may be found if the metric depends on internal coordinates 15. Specifically, the hierarchy emerges because the scales change throughout the internal dimensions, and the particle masses are literally a function of the location of our 3-brane in the internal space. In a very interesting work, Randall and Sundrum [15] have shown that even if there is only one extra dimension, and even if it remains very small, it may still be possible to obtain large mass hierarchies if the extra dimension is strongly curved. In their approach, this was provided by a large negative cosmological constant in the extra dimensions, which makes the metric a very sensitive function of the internal coordinates. Still, this bulk vacuum energy is cancelled on the brane by the brane cosmological constant, and so it would be cosmologically neutralized inside our world.

In this work we will explore the solutions where the bulk cosmological constant is not exactly cancelled by the brane one. Our motivation for this is twofold. First, we are interested in determining the circumstances under which it may be possible to have the brane universes which may be rapidly expanding, and hence lead to successful inflationary cosmology. Existence of such scenarios is very important in models with low compactification scale. In addition to the usual cosmological problems of the $4 D$ universe, there one has to explain the emptiness of large extra dimensions, which could contain far more energy than the brane. Since this would contradict the observations, such cosmological obstacles need to be answered dynamically, and inflation is the best candidate [1, 10, 12]. In the models with sub-millimeter internal dimension this problem is ably handled by inflation 
before stabilization of extra dimensions [12]. Second, it may be possible to find compactifications which are different from the example given in [15], but where nevertheless the mass hierarchy emerges because of the strongly curved internal dimensions. Indeed, we will present new solutions describing parallel branes. Each brane will have a small net cosmological constant. In some cases the net cosmological constant will be negative, which may be less attractive, but this possibility is not ruled out experimentally. The effective cosmological constant on each brane may be many orders of magnitude smaller than the natural scale due to the cancellation between the bulk cosmological constant and the brane tension. However, with the phenomenologically reasonable parameters needed to generate hierarchy, we find that the cancellation still falls short of producing an effective cosmological constant in the range of $10^{-120} M_{P l}^{4}$. Nevertheless, the mere fact that it may be possible to get a small cosmological constant from large ones in this way deserves notice. There may exist other solutions which are realized in a similar spirit, that yield an even smaller nonzero cosmological constant on the wall.

Throughout the article we will also discuss some issues of relevance for early cosmology and inflation on branes in curved backgrounds. Rather interestingly, many inflating branes get surrounded by event horizons in the bulk during inflation. As inflation nears the end, these horizons move slowly away from the brane. If the inflationary periods are long, the dynamics of the bulk event horizon may help solve the bulk horizon problem without significant fine tuning, since perturbations inside of the horizons get stretched and pushed far away (to the hidden wall?). Hence, such solutions may lead to economical higher-dimensional inflationary scenarios.

The paper is organized as follows. We begin by deriving the solutions corresponding to one and two branes in bulks with constant curvature. We then proceed to discuss the properties of brane spacetimes, discussing the generalization of the usual Friedmann equation in cosmology and the role of the bulk event horizons. In Section IV we consider the features of the new solution with two parallel branes, noting that the effective cosmological constant on each brane could be much smaller than the natural scale, although still too large to fit the observations. Finally, we close with the Conclusion. The Appendix contains details of the derivation of solutions.

\section{Solutions}

We begin by considering a $3 D$ domain wall, or a 3-brane, in a curved $5 D$ bulk, which couples gravitationally to the $5 D$ bulk theory. The action specifying the dynamics of the brane-bulk system is

$$
S=\int_{M} d^{5} x \sqrt{g}\left\{\frac{R}{2 \kappa_{5}^{2}}-\mathcal{L}_{\text {bulk }}\right\}-\int_{\partial M} d^{4} x \sqrt{g} \mathcal{L}_{\text {brane }}
$$

Our conventions are $R_{\nu \lambda \rho}^{\mu}=\partial_{\lambda} \Gamma_{\nu \rho}^{\mu}-\partial_{\rho} \Gamma_{\nu \lambda}^{\mu}+\ldots$, and $\eta_{\mu \nu}=\operatorname{diag}(-1,1,1,1,1)$, which are common in low energy string and M-theory. Further, we use $\kappa_{5}^{2}=\frac{8 \pi}{M_{*}^{3}}$, where $M_{*}$ is the 
$5 D$ Planck scale, roughly equal to the unification scale. The subscripts $M$ and $\partial M$ in the action stand for the $5 D$ bulk and the $4 D$ brane which from the standpoint of the bulk may be viewed as its boundary. We note that $\sqrt{g}$ in the $5 \mathrm{D}$ bulk and $4 \mathrm{D}$ boundaries are different, being related by $\sqrt{g}$ (boundary $)=\sqrt{g}($ bulk $) / \sqrt{g_{55}}$. This affects the equations of motion, ensuring that the projection of the brane's contribution to the bulk stress-energy tensor vanishes in the directions transverse to the brane.

To obtain the equations of motion, we can now vary the action (11) in the usual fashion. However, for the purposes of our investigation we can simplify our considerations without sacrificing physical contents. We are interested in determining the brane geometry at large distances when the bulk is curved, both during inflation on the brane(s) and in the limit when the expansion is very slow. Thus it is sufficient to consider solutions where the energy density on the brane is nearly constant, and so approximate the brane contribution to the stress-energy tensor by the brane tension. Further, recalling the properties of the scalar-tensor theories of gravity, which approximate the theory given by (1) in the infrared limit, when the bulk curvature is changing rapidly, generically the brane will neither inflate nor settle down to a highly homogeneous and isotropic state. Hence, there should be epochs when we can model the sources of curvature in the bulk by bulk cosmological terms. Therefore, the effective action which we consider is

$$
S=\int_{M} d^{5} x \sqrt{g}\left\{\frac{R}{2 \kappa_{5}^{2}}+\Lambda\right\}-\int_{\partial M} d^{4} x \sqrt{g} \sigma
$$

where we have taken the negative cosmological term in the bulk. Our motivation for this sign choice is that such models can be naturally obtained by compactifying supergravity theories in $10 D$ by using the Freund-Rubin ansatz [16], for example, compactifying the IIB string theory on $A d S_{5} \times S^{5}$ [17]. Other examples of domain walls in supergravity have been considered in [18, 19. A possibility that the world may look like a domain wall in the context of Hořava-Witten theory [7] has been pursued in [20], and possibilities for inflation in this model were studied in [21]. We have in mind a particular application of the solutions to the recent scenario proposed by Randall and Sundrum [15], and their solutions indeed emerge as the future asymptotics of some of the solutions presented below. However, all solutions will depend analytically on $\Lambda$, allowing one to continue $\Lambda$ to the range of negative values, corresponding to a positive bulk cosmological constant. The parameter $\sigma$ is the brane tension, which we will also allow to be both positive and negative (with $\sigma>0$ corresponding to positive brane tension).

The equations of motion which follow from (2) are

$$
R^{\mu}{ }_{\nu}-\frac{1}{2} \delta^{\mu}{ }_{\nu} R=-\kappa_{5}^{2} \frac{\sqrt{g_{\text {brane }}}}{\sqrt{g}} \sigma \delta(w) \operatorname{diag}(1,1,1,1,0)+\kappa_{5}^{2} \Lambda \delta^{\mu}{ }_{\nu}
$$

where the stress-energy tensor is a combination of wall and bulk terms. In general the ratio $\frac{\sqrt{g_{\text {brane }}}}{\sqrt{g}}$ cannot be gauged away. To find the specific form of the equations of motion, we need to write down the metric ansatz which will reflect the symmetries on the 3-brane. 
Here we can use some known solutions as a guideline. In the limit $\Lambda=0, \sigma>0$, the solution must correspond to the inflating 3-brane in $5 D$, found in [10]; it is given by $d s^{2}=\left(1-\frac{4 \pi \sigma}{3 M_{5}^{3}}|w|\right)^{2}\left(-d t^{2}+\exp \left(8 \pi \sigma t / 3 M_{5}^{3}\right) d \vec{x}^{2}\right)+d w^{2}$, and corresponds to a $3 D$ domain wall undergoing inflation, which is shrouded by a Rindler horizon in the bulk. In the limit $\Lambda=\kappa_{5}^{2} \sigma^{2} / 6$, the solution corresponds to a flat 3-brane in a $5 D A d S$ background, found in [15], and given by $d s^{2}=\exp \left(-\kappa_{5}^{2} \sigma|w| / 6\right)\left(-d t^{2}+d \vec{x}^{2}\right)+d w^{2}$. Hence we could look for a two-parameter family of solutions which interpolates between these limits, which would be de-Sitter on the wall and Anti-de-Sitter in the bulk. However, rather then restricting only to those $4 D$ space-time sections which are de-Sitter, we will merely require that they are maximally symmetric, thus representing any of the de Sitter, Anti-de-Sitter or Minkowski spacetimes in four dimensions. The metric ansatz which accomplishes this is

$$
d s_{5}^{2}=a^{2}(w)\left(-d t^{2}+e^{2 H t} d \vec{x}^{2}\right)+b^{2}(w) d w^{2}
$$

where the brane scale factor $H$ will be completely determined by the cosmological terms $\Lambda$ and $\sigma$ after we normalize the coefficient of $d t^{2}$ to unity on the brane. Indeed, if $H$ is imaginary, $H=i \mathcal{H}$, we can analytically continue the solution (雨) by a coordinate transformation $t=-i x^{\prime}, x=i t^{\prime}, y=y^{\prime}$ and $z=z^{\prime}$ to

$$
d s_{5}^{2}=a^{2}(w)\left(d x^{\prime 2}+e^{2 \mathcal{H} x^{\prime}}\left(-d t^{\prime 2}+d y^{\prime 2}+d z^{\prime 2}\right)\right)+b^{2}(w) d w^{2}
$$

a metric with $4 D$ Anti-de-Sitter slices as claimed. One must not ignore these solutions, since it is well known 22] that their slices correspond to open FRW universes, and hence are not ruled out by observations. In fact, for all cases where the brane metric is locally $A d S_{4}$, we will use the FRW representation

$$
d s_{5}^{2}=a^{2}(w)\left(-d t^{2}+|H|^{-2} \cos ^{2}(|H| t)\left(\frac{d r^{2}}{1+r^{2}}+r^{2} d \Omega\right)\right)+b^{2}(w) d w^{2}
$$

since we will have in mind cosmological evolution, where the solution (6) could be a reasonable approximation at times after inflation on the brane.

To solve the equations of motion, we can substitute the ansatz (41) into (3), and work out the details. Alternatively, we can work in the action, dimensionally reducing the theory to only one dimension, which is possible since the $4 D$ slices in (田) are maximally symmetric. The dependence of all geometric quantities on the coordinates of $4 D$ slices is completely prescribed by the symmetry. A straightforward calculation (see the appendix) gives the equations of motion, which in the gauge $b=1$ are

$$
\begin{aligned}
& a^{\prime 2}=H^{2}+\frac{\kappa_{5}^{2} \Lambda}{6} a^{2} \\
& a^{\prime \prime}-\frac{\kappa_{5}^{2} \Lambda}{6} a=-\frac{\kappa_{5}^{2}}{3} \sigma a(0) \delta(w)
\end{aligned}
$$

Again, we underline that $H^{2}$ can be both positive and negative, despite the slightly confusing notation. We will solve the equations for $H^{2}>0$, since the solutions for $H^{2}<0$ 
can be easily obtained by analytic continuation. Leaving the details for the appendix, when $H^{2}>0$ we can write the solution as

$$
a=\frac{6 H}{\kappa_{5} \sqrt{\kappa_{5}^{2} \sigma^{2}-6 \Lambda}}\left(\cosh \left(\sqrt{\frac{\kappa_{5}^{2} \Lambda}{6}} w\right)-\frac{\kappa_{5} \sigma}{\sqrt{6 \Lambda}} \sinh \left(\sqrt{\frac{\kappa_{5}^{2} \Lambda}{6}}|w|\right)\right)
$$

We see that at the location of the brane, $a(0)=\frac{6 H}{\kappa_{5} \sqrt{\kappa_{5}^{2} \sigma^{2}-6 \Lambda}}$. We therefore normalize the coordinates along the brane such that their coefficient is unity, which is accomplished by $t \rightarrow \kappa_{5} \frac{\sqrt{\kappa_{5}^{2} \sigma^{2}-6 \Lambda}}{6 H} t$, and $\vec{x} \rightarrow \kappa_{5} \frac{\sqrt{\kappa_{5}^{2} \sigma^{2}-6 \Lambda}}{6 H} \vec{x}$. Thus the expansion rate along the brane is $H=\kappa_{5} \sqrt{\kappa_{5}^{2} \sigma^{2}-6 \Lambda} / 6$, in comoving units. The normalized warp factor is

$$
a=\cosh \left(\sqrt{\frac{\kappa_{5}^{2} \Lambda}{6}} w\right)-\frac{\kappa_{5} \sigma}{\sqrt{6 \Lambda}} \sinh \left(\sqrt{\frac{\kappa_{5}^{2} \Lambda}{6}}|w|\right)
$$

Substituting the coordinate transformation into the metric we find

$$
d s_{5}^{2}=\left(\cosh \left(\sqrt{\frac{\kappa_{5}^{2} \Lambda}{6}} w\right)-\frac{\kappa_{5} \sigma}{\sqrt{6 \Lambda}} \sinh \left(\sqrt{\frac{\kappa_{5}^{2} \Lambda}{6}}|w|\right)\right)^{2}\left(-d t^{2}+e^{\kappa_{5} \sqrt{\kappa_{5}^{2} \sigma^{2}-6 \Lambda} t / 3} d \vec{x}^{2}\right)+d w^{2}
$$

This, finally, is our new solution for the case of a single brane. The warp factor is a combination of exponentials while the wall is curved. We will discuss its properties in the next section. Here we should point out one generic feature of wall models with localized energy density. Rather interestingly, the square of the rate of expansion on the brane, given by the Friedmann equation in the usual $4 D$ cosmology, is now

$$
H^{2}=\kappa_{5}^{2} \frac{\kappa_{5}^{2} \sigma^{2}-6 \Lambda}{36}
$$

This equation is a further extension of the generalized Friedmann equations of [23] because it incorporates bulk contributions in addition to the brane terms. When the bulk cosmological constant is zero, the expansion rate is given by the brane tension (and not its square). When the tension is zero, the expansion rate is equal to the square root of the bulk cosmological constant, i.e. just the usual Friedmann rule. In the general case when both terms are nonzero, the brane expansion rate is a hybrid of these two formulas. As we have announced above, for sufficiently large $\Lambda>\kappa_{5}^{2} \sigma^{2} / 6$ the "expansion rate" is imaginary, which means that the brane geometry is $A d S_{4}$ rather than $d S_{4}$. The imaginary numbers are removed by a proper analytical continuation, discussed above. We will return to more examples of single brane solutions in the next section.

Let us turn to solutions describing two branes. We will only consider solutions where the branes are perfectly parallel. Such solutions can be described by the action we have discussed above, which includes an additional contribution $\sim-\int_{\partial \bar{M}} d^{4} x \sqrt{g} \bar{\sigma}$. We will approach the problem by taking the solution (10) describing one brane in $A d S_{5}$ as a background, and will look for ways to fit the other brane in it, adjusting its tension 
as required. The backgrounds are warped products of the $4 D$ spacetime and the fifth dimension, implying that such geometries can be foliated by a congruence of slices which are conformal to the 3-branes. This allows us to find solutions by a simple pasting of the probe brane. Here we will briefly review the derivation, referring the reader to the appendix.

In order to match the background solution with the long range fields of the probe brane, and insure that the resulting array is a solution of the Einstein's equations, we will start with the generalized Friedmann equation (11) for the source brane together with the first of eq. (7). If we combine these two equations, we find

$$
a^{\prime 2}=\frac{\kappa_{5}^{4} \sigma^{2}}{36}-\frac{\kappa_{5}^{2} \Lambda}{6}+\frac{\kappa_{5}^{2} \Lambda}{6} a^{2}
$$

The RHS of this equation is not always positive definite, which will enable us to construct new solutions of parallel branes, different from the cases considered in [15]. This equation shows that both branes have the same intrinsic curvature, given by $(\sqrt{11})$, although their tensions are different. Away from the branes the original solution (9) solves (12) and the brane expansion rate is given by (11). This is just the gravitational field of the source brane. The second order differential equation in this case contains an additional Dirac $\delta$-function,

$$
a^{\prime \prime}-\frac{\kappa_{5}^{2} \Lambda}{6} a=-\frac{\kappa_{5}^{2}}{3} \sigma a(0) \delta(w)-\frac{\kappa_{5}^{2}}{3} \bar{\sigma} a\left(w_{c}\right) \delta\left(w-w_{c}\right)
$$

The effect of the branes is to compactify the bulk on the circle, $w \sim w+w_{c}$, and further orbifold the circle by $Z_{2}$ operation $w \sim-w$ in order to stabilize the solution. Substituting the solution (9) into (13), we obtain the matching condition

$$
\left(\sqrt{\frac{2 \kappa_{5}^{2} \Lambda}{3}}+\frac{\kappa_{5}^{3} \sigma \bar{\sigma}}{3 \sqrt{6 \Lambda}}\right) \sinh \left(\sqrt{\frac{\kappa_{5}^{2} \Lambda}{6}} w_{c}\right)=\frac{\kappa_{5}^{2}}{3}(\sigma+\bar{\sigma}) \cosh \left(\sqrt{\frac{\kappa_{5}^{2} \Lambda}{6}} w_{c}\right)
$$

The existence of solutions of this equation depends on the signs of the brane tensions $\sigma, \bar{\sigma}$ and the ratios $\frac{\sqrt{6 \Lambda}}{\kappa|\sigma|}, \frac{\sqrt{6 \Lambda}}{\kappa|\bar{\sigma}|}$, as discussed in the appendix. One can group those solutions into two main categories.

The first category of solutions is qualitatively new, since both branes have positive tension. For such solutions, the bulk cosmological constant must either be at least infinitesimally larger than the square of the larger of two tensions, which without loss of generality we can take to be the source brane, with tension $\sigma: 6 \Lambda>\kappa_{5}^{2} \sigma^{2}$, or infinitesimally smaller than the square of the smaller of two tensions, taken here as the probe brane tension: $6 \Lambda<\kappa_{5}^{2} \bar{\sigma}^{2}$. By (11), this means that the branes can never be completely flat unless they are infinitely far apart. Indeed, the branes are separated by a distance $w_{c}$ given by

$$
\tanh \left(\sqrt{\frac{\kappa_{5}^{2} \Lambda}{6}} w_{c}\right)=\frac{\kappa_{5} \sqrt{6 \Lambda}(\sigma+\bar{\sigma})}{6 \Lambda+\kappa_{5}^{2} \sigma \bar{\sigma}}
$$


and we see that if $6 \Lambda=\kappa_{5}^{2} \sigma^{2}, w_{c} \rightarrow \infty$. The warp factor on the probe brane is

$$
a\left(w_{c}\right)=\sqrt{\left|\frac{6 \Lambda-\kappa_{5}^{2} \sigma^{2}}{6 \Lambda-\kappa_{5}^{2} \bar{\sigma}^{2}}\right|}
$$

The equation for the warp factor is easily obtained from substituting the jump of the warp factor at the other brane $a^{\prime 2} / a^{2}\left(w_{c}\right)=\kappa_{5}^{4} \bar{\sigma}^{2} / 36$ into eq. (12). Clearly, the warp factor on the brane with smaller tension is smaller. Hence this brane should be the candidate for our world, since the warping will lower the scales there. The amount of warping and the brane separation are completely specified by the values of the cosmological terms, which as we will see below is a generic property of the parallel brane configurations. Hence this obviously suggests an intricate relationship between the cosmological constant problem and the mass hierarchy. We will return to this interesting relationship later. When $6 \Lambda>\kappa_{5}^{2} \sigma^{2}$, since both branes feel a net negative curvature, they may be viewed as FRW $A d S_{4}$ universes. The metric of the complete solution can therefore be written as

$$
d s_{5}^{2}=a^{2}(w)\left(-d t^{2}+\frac{36}{6 \kappa_{5}^{2} \Lambda-\kappa_{5}^{4} \sigma^{2}} \cos ^{2}\left(\frac{\kappa_{5} \sqrt{6 \Lambda-\kappa_{5}^{2} \sigma^{2}}}{6} t\right)\left(\frac{d r^{2}}{1+r^{2}}+r^{2} d \Omega\right)\right)+b^{2}(w) d w^{2}
$$

where $a(w)$ is given by (9) with additional identification in the bulk $w \sim w+w_{c}, w \sim-w$. In the case $6 \Lambda<\kappa_{5}^{2} \bar{\sigma}^{2}$, the longitudinal metric is replaced by the de-Sitter one. The solution for $a(w)$ for $w<0$ is therefore a mirror image of the solution for $w>0$ around the vertical axis at $w=0$, as it should be for orbifold constructions. Note that $a(w)$ is cusping upwards at both branes, because both branes have positive tension. This is possible because the solution (9) for $a$ bounces in the bulk between the two branes, as can be seen from the eq. (12). The RHS can vanish between the branes, and $a$ can reexpand again. This of course is induced by the negative curvature on the branes. We will discuss phenomenological aspects of these solutions later.

The second category is comprised of the solutions where one of the branes has a negative tension, whereas the other has a positive tension. The solutions however fall into three very different subcategories. In the first case, if $\sqrt{\Lambda}=\frac{\kappa_{5} \sigma}{\sqrt{6}}=-\frac{\kappa_{5} \bar{\sigma}}{\sqrt{6}}$, the distance between the branes $w_{c}$ is arbitrary, as well as the amount of warping at the brane with negative tension (we remind the reader that we have normalized the warp factor to unity at the source brane). This is the solution given in [15], describing two flat branes. In the other subfamily of solutions, if the absolute value of the negative tension is even slightly larger than the positive tension, which is possible if the bulk cosmological constant is slightly smaller than the square of the positive tension (which implies that both branes are inflating, as can be seen from (11)), the distance between the branes is not arbitrary any more, but is completely specified by the cosmological terms:

$$
\tanh \left(\sqrt{\frac{\kappa_{5}^{2} \Lambda}{6}} w_{c}\right)=\frac{\kappa_{5} \sqrt{6 \Lambda}(|\bar{\sigma}|-\sigma)}{\kappa_{5}^{2} \sigma|\bar{\sigma}|-6 \Lambda}
$$


Obviously, $w_{c}$ could be arbitrary only in the simultaneous limit $\sigma=|\bar{\sigma}|=\frac{\sqrt{6 \Lambda}}{\kappa_{5}}$. The warp factor at the other brane is

$$
a\left(w_{c}\right)=\sqrt{\frac{\kappa_{5}^{2} \sigma^{2}-6 \Lambda}{\kappa_{5}^{2}|\bar{\sigma}|^{2}-6 \Lambda}}
$$

The warp factor at the brane with negative tension is smaller than unity, which is qualitatively the same as in the solution of [15]. Unlike there, however, the amount of warping is completely specified once the cosmological terms are given. The explicit form of the solutions is locally the same as (4), with the warp factor given by (9) and with identifications $w \sim w+w_{c}, w \sim-w$. The warp factor cusps upwards on the positive tension brane, and downwards on the negative tension brane. The third subfamily of solutions has the positive tension of one brane larger than the absolute value of the negative tension of the other brane. The bulk cosmological constant must be larger than the square of the positive tension. Again, even if the differences between the cosmological terms are infinitesimal, the distance between the branes is completely specified once the cosmological terms are known:

$$
\tanh \left(\sqrt{\frac{\kappa_{5}^{2} \Lambda}{6}} w_{c}\right)=\frac{\kappa_{5} \sqrt{6 \Lambda}(\sigma-|\bar{\sigma}|)}{6 \Lambda-\kappa_{5}^{2} \sigma|\bar{\sigma}|}
$$

Both branes feel a net negative curvature, however, and hence the metric of the solution is formally the same as (17), with different values of the cosmological terms. The warp factor $a$ again cusps upwards on the positive tension brane and downwards on the negative tension brane. However, the warp factor at the negative tension brane is profoundly different from the previous two cases: it is much larger there than on the brane with the positive tension. Its value is also uniquely specified by the cosmological terms:

$$
a\left(w_{c}\right)=\sqrt{\frac{6 \Lambda-\kappa_{5}^{2} \sigma^{2}}{6 \Lambda-\kappa_{5}^{2}|\bar{\sigma}|^{2}}}
$$

This can be understood as follows: this warp factor is in fact a negative of the actual solution of the Einstein's equations. Since the physics on either brane does not know about the sign of $a$, we could simply ignore it, and instead write just the absolute value of $a$. Then the flow of the warp factor is opposite to the previous example, and hence the magnitude of $a$ is naturally greater at the brane with negative tension.

The latter two subfamilies of solutions suggest that the construction of [15] satisfying the condition $\sigma=|\bar{\sigma}|=\frac{\sqrt{6 \Lambda}}{\kappa_{5}}$ may be unstable to small perturbations of the brane tensions. Even a small mismatch between the brane tensions and the bulk cosmological constant could offset the necessary amount of warping, $a\left(w_{c}\right) \sim 10^{-15}$, regardless of where the perturbation takes the solution. However, once solutions belong to either of the two subfamilies with mismatch already present, their stability under small perturbations is restored. We will consider some explicit examples later.

There also exist solutions of two parallel branes when $\Lambda<0$ (positive cosmological constant). This can be seen from (14) and the fact that the hyperbolic functions should 
be replaced by trigonometric ones. These solutions can be useful as toy models for inflationary model building. Here we will list the solutions with their principal properties, as we did for the case when $\Lambda>0$. We leave the details for the appendix, and a brief discussion of phenomenology of such solutions for the Sec. IV.

All the solutions with $\Lambda<0$ represent two parallel inflating branes separated by some bulk distance in the orbifold construction. Using (11), we see that the expansion rate is given by $H^{2}=\kappa^{2} \frac{\kappa^{2} \sigma^{2}+6|\Lambda|}{36}$, and there is a net positive cosmological constant on each brane. The metric of the complete solution is

$$
d s_{5}^{2}=\left(\cos \left(\sqrt{\frac{\kappa_{5}^{2}|\Lambda|}{6}} w\right)-\frac{\kappa_{5} \sigma}{\sqrt{6|\Lambda|}} \sin \left(\sqrt{\frac{\kappa_{5}^{2}|\Lambda|}{6}}|w|\right)\right)^{2}\left(-d t^{2}+e^{\kappa_{5} \sqrt{\kappa_{5}^{2} \sigma^{2}+6|\Lambda|} t / 3} d \vec{x}^{2}\right)+d w^{2}
$$

with the orbifold conditions $w \sim w+w_{c}$ and $w \sim-w$. The solutions are now divided into three categories. There are two rather special cases, and the first is defined by $\bar{\sigma}=-\sigma$. It corresponds to two branes with tensions of the opposite sign. Both of these branes are inflating, as should be expected from (11) and $\Lambda<0$. The interbrane distance is still fixed, and is given by

$$
w_{c}=\sqrt{\frac{6}{\kappa_{5}^{2}|\Lambda|} \pi}
$$

Having normalized the warp factor to unity on the positive tension brane (source brane), we find that at the negative tension brane it is just $a\left(w_{c}\right)=-1$. Hence in this particular case, the mass scales on both branes coincide. The second special case has both tensions positive. However, the bulk cosmological constant is given by the product of the tensions, $6|\Lambda|=\kappa_{5}^{2} \sigma \bar{\sigma}$. In this case, the interbrane distance is

$$
w_{c}=\sqrt{\frac{3}{2 \kappa_{5}^{2}|\Lambda|} \pi}
$$

while the warp factor is

$$
a\left(w_{c}\right)=-\frac{\kappa_{5} \sigma}{\sqrt{6|\Lambda|}}
$$

Despite the fact that both branes have positive tension, there is still a horizon between them. The reason is that the brane with larger tension is more repulsive than the one with smaller tension, and that the bulk cosmological constant is positive. The last category of solutions is the most generic, and includes all those configurations for which $\sigma+\bar{\sigma} \neq 0$ and $6|\Lambda| \neq \kappa_{5}^{2} \sigma \bar{\sigma}$. Therefore, the solutions satisfy

$$
\tan \left(\sqrt{\frac{\kappa_{5}^{2}|\Lambda|}{6}} w_{c}\right)=\frac{\kappa_{5} \sqrt{6|\Lambda|}(\sigma+\bar{\sigma})}{\kappa_{5}^{2} \sigma \bar{\sigma}-6|\Lambda|}
$$

wherefore we can determine the interbrane distance. It is given by

$$
w_{c}=\sqrt{\frac{6}{\kappa_{5}^{2}|\Lambda|}}\left(\tan ^{-1}\left(\frac{\kappa_{5} \sqrt{6|\Lambda|}(\sigma+\bar{\sigma})}{\kappa_{5}^{2} \sigma \bar{\sigma}-6|\Lambda|}\right)+2 n \pi\right)
$$


where the contribution $2 n \pi$ takes into account the periodicity of the tangent function. The warp factor is then given by

$$
a\left(w_{c}\right)=\zeta \sqrt{\frac{6|\Lambda|+\kappa_{5}^{2} \sigma^{2}}{6|\Lambda|+\kappa_{5}^{2} \bar{\sigma}^{2}}}
$$

where $\zeta=-\operatorname{sgn}\left\{\cos \left(\sqrt{\frac{\kappa_{5}^{2}|\Lambda|}{6}} w_{c}\right)\right\}$. Thus, there may or may not be a horizon between the branes, depending on the interplay of the three terms $\sigma, \bar{\sigma}$ and $|\Lambda|$. The distance between the branes is however controlled by the scale of the bulk cosmological constant, $L \sim \sqrt{\frac{6}{\kappa_{5}^{2}|\Lambda|}}$, since the inverse tangent function is always bounded by unity. Hence in a de Sitter background the scale of the interbrane distance is only weakly affected by the bulk geometry, unlike in the $A d S$ background. We will discuss these solutions further in Sec. IV.

\section{Isolated Domain Walls}

We now examine the solutions (10) in more detail. Let us begin with $\Lambda>0$ (which corresponds to negative bulk cosmological constant) and $\sigma>0$ (positive brane tension). In the limit $\Lambda \rightarrow 0$ we recover precisely the inflating 3 -brane solution of [10, which generalizes the inflating domain walls [24] in $4 D$ space-time. In the limit $6 \Lambda=\kappa_{5}^{2} \sigma^{2}$, we get the Minkowski 3-branes in $A d S_{5}$ background, found by Randall and Sundrum [15]. For any value of $\Lambda$ between these two extremes, $0 \leq \Lambda \leq \frac{\kappa_{5}^{2} \sigma^{2}}{6}$, we find inflating 3-branes in $A d S_{5}$ backgrounds.

Another notable property of the solutions with $0 \leq \Lambda \leq \frac{\kappa_{5}^{2} \sigma^{2}}{6}$ is the existence and location of the bulk event horizon. As can be seen from (10), the 3-brane is surrounded with a Rindler-type event horizon in the bulk, which is located at the hypersurface $w=$ const where the metric coefficient $g_{00}$ vanishes. A simple calculation shows that this is at

$$
w_{H}=\sqrt{\frac{3}{2 \kappa_{5}^{2} \Lambda}} \ln \left(\frac{\kappa_{5} \sigma+\sqrt{6 \Lambda}}{\kappa_{5} \sigma-\sqrt{6 \Lambda}}\right)
$$

In the limit $\Lambda \rightarrow 0$, this reproduces $w_{H}=\frac{3 M_{5}^{3}}{4 \pi \sigma}$, found in [10] for an inflating 3-brane in a flat bulk. On the other hand, when $\Lambda \rightarrow \kappa_{5}^{2} \sigma^{2} / 6, w_{H}$ diverges. The presence of the Rindler horizon in the bulk could be helpful because of the cosmic no-hair theorem 25], which has two aspects in this context. First, it pushes any bulk perturbations which occur near the brane away from it. Second, it prevents perturbations at distances larger than the Rindler horizon from ever affecting the brane. From the vantage point of the brane those perturbations behave as if they have fallen inside of a black hole. As a result, any perturbations at distances shorter than the Rindler horizon are pushed outside of it, while the perturbations at longer distances are harmless to begin with. On the other 
hand, as the brane expansion rate $H=\kappa_{5} \frac{\sqrt{\kappa_{5}^{2} \sigma^{2}-6 \Lambda}}{6}$ decreases (which can be accomplished either by increasing the bulk cosmological constant $\Lambda$ or by decreasing the brane tension $\sigma)$, the Rindler horizon moves farther and farther from the brane. Hence if the bulk was initially homogeneous over distances $\sim \frac{3 M_{5}^{3}}{4 \pi \sigma}$, it may become homogeneous over ever larger distances as $H$ approaches zero. To see when this may happen, we should note that the time it takes for perturbations near the brane to get near the event horizon is roughly given by $\tau \sim w_{H}$. Hence to fully benefit from the presence of the Rindler horizon in the bulk during inflation on the wall, the expansion rate should decrease slowly, at timescales at least of order of $w_{H}$ or larger. Otherwise the horizon would move away from the brane faster than the perturbations, trapping them forever near the brane. Therefore we find a fundamental criterion for the slow roll condition for inflation on the brane, which should be satisfied to simultaneously resolve both the brane and the bulk homogeneity problem without fine tuning: the expansion rate on the brane should change at timescales at least as large as $w_{H}$. This is effectively similar to having asymmetric inflation of [12].

As long as the Poincare symmetry on the 3-brane is unbroken, the range of parameters for which $\Lambda>\kappa_{5}^{2} \sigma^{2} / 6$ cannot be reached by the rolling of the cosmological terms. This is because for $\Lambda>\kappa_{5}^{2} \sigma^{2} / 6$, the expansion rate on the brane is imaginary, and so the intrinsic geometry of the brane is $A d S_{4}$, as can be seen from (11). We have seen that our new solution with two branes (17) is precisely of this form. Indeed, this should be expected since if the brane tension $\sigma$ vanishes, the brane must bend to conform to the bulk $A d S_{5}$ geometry, becoming just an $A d S_{4}$ slice of it. The brane tension $\sigma$ resists this, and the favored ground state of the system depends on the rigidity of the brane relative to the extrinsic curvature imposed by the bulk. We will not study the mechanisms which can break the Poincare symmetry on the brane, but will merely assume that solutions with a definitive sign of $H^{2}$ are at least locally minima of the Hamiltonian, and are perturbatively separated from the solutions with the opposite sign of $H^{2}$. However, it would be interesting to investigate this in more detail. We note that the hyperbolic functions in $g_{00}$ of (10) remain unchanged. The location of the Rindler horizon (29) gets pushed beyond infinity, in a manner of speaking, because $g_{00}$ never vanishes for any value of $w$.

What happens if $\Lambda<0$ ? As we have already discussed in the previous section for the case of two parallel branes, this corresponds to the positive bulk cosmological constant, in our conventions, leading to an even more vigorous inflation on 3-branes than when $\Lambda>0$. Indeed, this remains true for a single brane case too. The solution is formally the same as (22), except that the only identification is $w \sim-w$, and otherwise $w$ is unbounded. The expansion rate along the brane is now increased by the bulk contributions, as expected. The metric however has become periodic in the bulk, including the periodic distribution of the Rindler horizons which appear at distances $w_{H}+n \pi \sqrt{\frac{6}{\kappa_{5}^{2}|\Lambda|}}$, where the fundamental distance $w_{H}$ is given by

$$
w_{H}=\sqrt{\frac{6}{\kappa_{5}^{2}|\Lambda|}} \tan ^{-1}\left(\frac{\sqrt{6}|\Lambda|}{\kappa_{5} \sigma}\right)
$$

A curious property of this formula is that the location of the Rindler horizon adjacent to 
the brane oscillates as a function of $\frac{\sqrt{|\Lambda|}}{\sigma}$, and can be arbitrarily close and far from the brane.

Now we consider the solutions for the brane with a negative tension, $\sigma=-|\sigma|$. When $\Lambda>0$, the solutions are still given by (10). Rather peculiarly, the intrinsic geometry on the brane is still inflating, even though the tension is negative, as can be seen clearly from the generalized Friedmann equation (11). However, the difference appears in the absence of the Rindler horizon in the bulk. The coefficient $g_{00}$ does not vanish at any hypersurface $w=$ const. In contrast to the case $\sigma>0, \Lambda>\kappa_{5}^{2} \sigma^{2} / 6$, the brane is now attractive for the probes in the bulk, rather than repulsive. This means that any object which may be present in the bulk will fall onto the brane in a finite amount of time. Such a rain of particles from extra dimensions further underscores the instability of the solution and may impose strong phenomenological constraints on model building. It would be of interest to investigate it in more detail.

The negative tension branes appear generically even more unstable if we take $\Lambda<0$. The solution is given by (22), but without the periodic identification $w \sim w+w_{c}$. The brane is still attractive for the particles in the bulk. However, now it is surrounded by a repulsive Rindler horizon, which limits the causal influence of the brane on the bulk physics. Hence the phenomena on the brane could exert influence in the bulk up to horizon distances, but not beyond. This means that outside of the horizon, there may be, for example, a highly inhomogeneous distribution of particles which will all however fall on the brane in some finite time, and possibly destroy any homogeneity produced by inflation. Clearly, such a situation would be devastating. However, as we know, in this case the distance of the horizon oscillates, and can be arbitrarily large. One may therefore imagine some physics on the brane reaching out into the bulk to homogenize it during such phases. Determining the possibility or impossibility of such phenomena would obviously require scrutiny beyond the scope of the present investigation, and we will not address it further.

\section{Parallel Walls}

We now return to the solutions which describe two parallel branes. Our main interest is to explore the new family where both branes have positive tension (17), but each have FRW $A d S_{4}$ geometry. The branes must be negatively curved because the effective cosmological constant felt on each brane is not completely cancelled. This is acceptable in principle if the curvature is small, even if negative. The $A d S_{4}$ is crucial for the existence of this solution, since it enables the warp factor (9) to bounce between the branes, which can be seen from eq. (12). However, we will also reflect on the solutions where one wall has positive tension and another negative. In particular, the example where the branes have a small net positive curvature is quite interesting, since both branes are simultaneously undergoing inflation. 
The bulk geometry in the direction orthogonal to the brane is more complex than in the example of the two brane solution of [15]. The deviation of the warp factor given by (9) takes into account the bending of the branes in the bulk. Still, we can follow closely the proposal of [15], which posits that a physical particle living on either brane couples to only the $4 D$ conformal metric on each brane, while the warp factor only renormalizes the physical scales of the theory. However, an additional constraint must be considered. Since in general there is an excess cosmological constant on each brane, we must ensure that the numbers needed to produce the $M_{P l}-m_{E W}$ hierarchy do not give an excessively large leftover cosmological constant on the brane. As we have suggested before, this means that there is a very intricate relationship between the cosmological constant problem and the hierarchy problem. One must therefore use these extended criteria to check the phenomenological feasibility of the constructions based on parallel branes. In general, we will see that this is not satisfied in the simple examples, since the numbers needed to produce mass hierarchy fall short of cancelling the cosmological constant to experimentally permitted values. Nevertheless, for a certain range of admissible parameters which do lead to the correct mass hierarchy, there is a very significant reduction of the effective cosmological constant on the branes. In a sense the picture which emerges is a hybrid of the ideas of [1] and [15]. To reduce the net cosmological constant on the brane, the unification scale should be lower than the Planck scale and the bulk should be large. So a part of the hierarchy is generated by raising the $4 D$ Planck scale as in [1]. The warping then produces additional contribution to the mass hierarchy, lowering the fundamental scale down to $m_{E W}$. Therefore although this mechanism does not yet solve the cosmological problem, it is still quite interesting. It gives a possibility to have a very small but nonzero cosmological constant on the brane. There may exist other solutions describing two branes, with some additional scalars in the bulk (such as the moduli fields in string theory) which could produce a more complicated running of the warp in the bulk, yielding a more efficient cancellation of the cosmological constant on our wall. Such scenarios may be useful for understanding why the cosmological constant may be very small but nonvanishing.

Since the scale of the particle dynamics varies non-monotonically in the bulk, the hierarchy would have to come from the ratio of the warp factors at different branes. Having normalized one of them to unity, we can simply use the value of the other as given in eq. (16). Then we recall the relationship between the fundamental scale, given by $M_{*}$ and the Planck scale $M_{P l}$. Since $\kappa_{5}^{2}=\frac{8 \pi}{M_{*}^{3}}$ and $\kappa_{P}^{2}=\frac{8 \pi}{M_{P l}^{2}}$, using the action (1) one finds that 15

$$
\frac{1}{2 \kappa_{P}^{2}}=\frac{1}{2 \kappa_{5}^{2}} \int_{0}^{1} d \vartheta w_{c} a^{2}\left(w_{c} \vartheta\right)
$$

Our wall should be the one with smaller warp factor, therefore it is parameterized by $\bar{\sigma}$. The net cosmological constant on our wall must be very small. The net cosmological constant on the walls is a mismatch between the bulk cosmological constant and $\sigma^{2}$, as can be seen from (11): $|H|^{2}=\kappa_{5}^{2} \frac{6 \Lambda-\kappa_{5}^{2} \sigma^{2}}{36}=M_{P l}^{2} \epsilon$ where $\epsilon=\frac{\lambda}{M_{P l}^{4}}$ is a small dimensionless parameter. 
Using (15), we can get the distance between the branes in terms of the leftover cosmological constant $\epsilon$. To the lowest order, we can use the formula (15) and $\sqrt{\frac{\kappa_{5}^{2} \Lambda}{6}} w_{c} \sim \frac{\kappa_{5}^{2} \sigma}{6} w_{c}$. Numerically, this quantity must be large in order to match the small $\epsilon$. After a little algebra, we find

$$
e^{-\frac{\kappa_{5}^{2} \sigma}{6} w_{c}}=\left(\frac{9 M_{P l}^{2} \epsilon}{\kappa_{5}^{4} \sigma^{2}}\right)^{1 / 2}
$$

This formula gives the distance between the branes. When we substitute the formula for the warp factor (9) into (31), evaluate the integral and use $\epsilon<<1$, we find that

$$
\kappa_{P}^{2}=\frac{\kappa_{5}^{4} \sigma}{3}
$$

From this equation, we can express $\sigma$ as a function of $M_{*}$ and $M_{P l}$, getting $\sigma=\frac{3 M_{*}^{6}}{8 \pi M_{P l}^{2}}$. Next, it is easy to check that the hierarchy constraint is

$$
a\left(w_{c}\right)=\frac{m_{E W}}{M_{*}}
$$

Evaluating $a\left(w_{c}\right)$ we find $a\left(w_{c}\right) \sim 2 \sqrt{\epsilon}\left(\frac{M_{P l}}{M_{*}}\right)^{3}$. Now we can finally consider the numerical relationships. Our equations translate to the following formulas for $\epsilon, \bar{\sigma}$ and $\sigma$ in terms of the mass scales $M_{*}, M_{P l}, m_{E W}$ :

$$
\begin{aligned}
\epsilon & =\frac{m_{E W}^{2} M_{*}^{4}}{4 M_{P l}^{6}} \\
\bar{\sigma} & =\frac{3 m_{E W} M_{*}^{5}}{8 \pi M_{P l}^{2}} \\
\sigma & =\frac{3 M_{*}^{6}}{8 \pi M_{P l}^{2}}
\end{aligned}
$$

The distance between the branes is

$$
w_{c}=\left(\frac{M_{P l}}{M_{*}}\right)^{2} M_{*}^{-1} \ln \left(\frac{4 M_{*}^{2}}{m_{E W}^{2}}\right)
$$

These equations show that in addition to the mass hierarchy there also arises an interesting hierarchy of the cosmological constants: the residual cosmological constant $\lambda$ is smaller from the tension of our wall $\bar{\sigma}$, which in turn is smaller from the tension of the other wall $\sigma$. The cosmological terms increase by factors of $\frac{m_{E W}}{M_{*}}$. All the while, the distance between the branes may be much larger than the unification length $M_{*}^{-1}$. In fact, even this fact is a confirmation of the induced hierarchy of cosmological constants: the separation between branes is a bulk phenomenon, controlled by the bulk scale $\sigma^{1 / 4}$, while the phenomena on the brane are controlled by $\lambda^{1 / 4}$. Hence the situation indeed represents an interesting combination of elements of [1] and [15]. However, one can verify that there is a lower bound on $\lambda, \lambda \geq 10^{-32}(\mathrm{TeV})^{4}$, which is saturated when $M_{*} \sim \mathrm{TeV}$. This is too large by about 30 orders of magnitude. Also, in this case $\sigma \sim \bar{\sigma} \sim \lambda$, and the smallness of $\lambda$ becomes 
just the usual fine tuning. The distance between the branes would then be $w_{c} \sim 10^{15} \mathrm{~cm}$. Nevertheless, generically $\lambda$ may still be considerably smaller than the natural scale set by the brane tension on the hidden wall. This reduction of the cosmological constant is purely geometrical. Since the brane dwells in the curved bulk, when it is located in the region of smaller bulk curvature, it will bend less. The amount of bending measures the effective cosmological constant on the wall, and is determined by the location of the brane, which is controlled by the interactions of the brane with the bulk and the hidden brane. Hence the bulk geometry needed to produce the hierarchy of particle physics scales could be useful to reduce the severity of the fine tuning of cosmological constant.

The warp factor for the solutions with one positive tension brane and one negative tension brane is very similar to the case when both branes have positive tension. Thus it is clear that as long as the warp factor is very small on the brane with negative tension and $\kappa_{5}^{2} \sigma^{2}=6 \Lambda-\frac{36 M_{P l}^{2}}{\kappa_{5}^{2}} \epsilon \sim 6 \Lambda$, the equations (35) and (36) would remain correct, now corresponding to a positive cosmological constant $\lambda=\epsilon M_{P l}^{4}$ on our wall. However, if we substitute for the scale $M_{*} \sim M_{P l}$, as has been suggested in [15, we would get $\lambda \sim 10^{-32} M_{P l}^{4}$, i.e. a very large net cosmological constant.

In the case of solutions with one positive tension brane and one negative tension brane, there exists another possibility for getting a tiny $\lambda$. Suppose that the bulk cosmological constant is very small, $6 \Lambda<<\kappa_{5}^{2} \sigma^{2}$. Then if $\lambda=H^{2} M_{P l}^{2}$ is taken as the usual net cosmological constant on the wall, from (11) if $\sigma / M_{*}^{4}<1$, also $\sigma^{2} / M_{*}^{8}<<1$. Because this combination contributes to $\lambda$, it may be possible to make $\lambda$ very small. However, as can be seen from (31), in this case $\Lambda=\frac{3 M_{*}^{9}}{4 \pi M_{P l}^{4}}$. From $H^{2}=\epsilon M_{P l}^{2}$ and $6 \Lambda<<\kappa_{5}^{2} \sigma^{2}$ we get $\sigma=\frac{3}{4 \pi} \sqrt{\epsilon} M_{*}^{3} M_{P l}$. Therefore the condition $6 \Lambda<<\kappa_{5}^{2} \sigma^{2}$ translates into $\frac{M_{*}}{M_{P l}}<<\epsilon^{1 / 6}$. Getting $\epsilon$ to be small enough requires $M_{*}$ too small, $M_{*} \leq 10^{-20} M_{P l} \sim 100 \mathrm{MeV}$. Strictly speaking, these arguments show that the special solution with two flat branes and $6 \Lambda=$ $\kappa_{5}^{2} \sigma^{2}=\kappa_{5}^{2} \bar{\sigma}^{2}$ [15 is unstable in the sense that even a small mismatch between the bulk and brane terms will leave too large a cosmological constant on our wall.

The solutions with $\Lambda<0$ are rather different from those with $\Lambda>0$. If we consider the integral (31), for a large range of parameters it is well approximated by

$$
w_{c} \int_{0}^{1} d \vartheta a^{2}\left(w_{c} \vartheta\right) \sim\left(1+\frac{\kappa_{5}^{2} \sigma^{2}}{6|\Lambda|}\right) w_{c}
$$

This is because the warp factor is now oscillatory. Hence, the reduced Planck scale is (up to factors of order unity)

$$
\kappa_{P}^{2} \approx \frac{6 \kappa_{5}^{2}|\Lambda|}{\left(6|\Lambda|+\kappa_{5}^{2} \sigma^{2}\right) w_{c}}
$$

Using $H^{2}=\frac{\lambda}{M_{P l}^{2}}$, we can determine the net cosmological constant on the walls in this limit. It is

$$
\lambda=\frac{4 \pi}{3} \frac{|\Lambda|}{M_{*}^{6} w_{c}} M_{P l}^{4}
$$


Now, to put our solutions in context, we recall that if the theory is really unified at the scale $M_{*}$, then the bulk cosmological constant cannot exceed it by much. Hence, $\Lambda \leq M_{*}^{5}$. Then requiring that $\lambda$ is within the observationally allowed range would require

$w_{c} \geq 10^{120} \frac{|\Lambda|}{M_{*}^{5}} M_{*}^{-1}$. Because of the periodicity of the distance formula (27), this equation can be satisfied in principle. But from (38), taking the natural scale of the tension not to exceed $M_{*}$ as well, we get $M_{P l}^{2} \sim w_{c} M_{*}^{3}$. Since $M_{*} \geq 1 T e V$, the distance between the branes will reduce the net cosmological constant by at most 32 orders of magnitude, when $M_{*}$ is exactly a $\mathrm{TeV}$. Hence again the net cosmological constant on the wall is bounded from below by $\lambda \geq 10^{-32}(\mathrm{TeV})^{4}$, unless the bulk cosmological constant is fine-tuned to an extremely low value. However, the formula for the warp factor (28) shows that in such a case further fine tuning of the wall tensions would be required to produce the mass hierarchy.

However, we again stress that it is important to note that although the parallel brane solutions discussed here only partially succeed in simultaneously resolving the mass hierarchy problem and reducing the cosmological constant fine tuning problem, the decrease in the net cosmological term can be nonnegligible. This suggests interesting possibilities. It may happen that there exist parallel brane solutions similar to (17) but with a different behavior of the bulk energy density, and different brane tensions, which could give more efficient screening of the cosmological constant on our wall. For example, such solutions could be realized in presence of additional bulk scalars, which can further modify the "running" of the cosmological terms in the bulk, or it may happen that the cancellation can be augmented in the presence of more large internal dimensions. We must note here however that the cosmological constant problem in a universe comprising of a single brane need not be as closely related to the mass hierarchy, since if a brane is alone in the universe, the cosmological constant on it can be tuned to an arbitrary value. Thus the cosmological constant problem acquires its more conventional guise in the lone brane models.

\section{Conclusions}

It is widely believed that the unified theories of interactions which contain quantum gravity should have more than four space-time dimensions. Contrasting the conventional approach to additional dimensions which are curled up at extremely short distances, the recent proposal for explaining the hierarchy problem envisions a more active role of the extra dimensions. The suggestion that there may be large sub-millimeter dimensions 11 has opened up a new alley of model-building, with many interesting applications in particle physics. The understanding of cosmology in such models is still at an early stage. There are several proposals for the description of the early universe which may give a picture that can conform with the standard Big Bang cosmology at low temperatures 11, 12. However, it is also possible that the extra dimensions may play other roles, complementing the proposal of [1] as discussed in [15]. 
Here we have taken the view that the extra dimensions could play a role which combines the proposals of [1] and [15]. This view was illustrated by deriving new large families of solutions of Einstein's equations that describe single 3-branes and pairs of 3-branes, which are located in a curved $5 D$ world, and may themselves also be curved. Since the intrinsic curvature of the walls amounts to a net cosmological constant as viewed by an observer pinned to the wall, it is important to check that the numerical values of parameters in models with hierarchy do not give an excessively large cosmological constant. For solutions which represent individual 3-branes, the net cosmological constant can always be tuned down to an arbitrarily small value. Thus, while these solutions are safe, they do not tell anything about the cosmological constant problem.

For the solutions of two parallel 3-branes, the situation is more interesting. The hierarchy in these solutions emerges because two walls are located at different places in the curved bulk. Hence the variation of the metric in the bulk from one wall to another may renormalize the microphysical scales. As a result, the walls will generically have to bend in order to fit in the bulk geometry. The amount of bending of each wall depends on its location, and the cosmological constant on the wall is proportional to its net curvature. Therefore, there will be a natural hierarchy of cosmological constants in the solutions. We have seen that the net cosmological constant on our wall may become many orders of magnitude smaller than its naturally expected magnitude. However in the simple solutions we have considered, this geometric softening of the cosmological constant is not sufficiently strong to bring it down to $10^{-120} M_{P l}^{4}$. Nevertheless, the solutions show a tendency to produce a net cosmological constant on our wall which can be much lower than expected, while still keeping the true wall tension large. Perhaps there exist different solutions representing curved walls where the screening of the cosmological constant on our wall is more efficient.

We underline however that even if the mechanism were successful, it would not have represented a full solution to the cosmological constant problem. Rather, it would still need some amount of tuning, such as explaining why exactly our wall would be located at a place in the bulk where the bulk curvature would bend it by a right amount. But this question may be related to the problem of moduli stabilization, and hence beyond the scope of the formalism pursued here. However, if one assumes that the moduli can be stabilized, then the location of the brane in the bulk may help explain why cosmological constant would be small but nonzero.

The cosmology of models with dynamical extra dimensions is still at a very early stage, and various investigations have been done so far [6, 10, 11, 12, 20, 21, 23, 26, 27, 32, 33, 34]. Here we have considered a simple effective description of domain walls in $5 D$, and have found that among the curved domain walls there is a profusion of those which are inflating in the longitudinal directions. But before such solutions can be viewed as candidates for realistic models of brane cosmology, it must be possible for inflation on the brane to end, accomplishing many set requirements, such as producing enough density perturbations and sufficient reheating. Here we will briefly remark on some basic features of inflationary 
model building in the braneworld framework. The examples of inflating walls show that the wall inflation, driven by some conventional inflationary model on the wall which controls the evolution when the bulk physics is weakly coupled, could also help with the horizon problem in the bulk [10]. A conventional inflation produces a positive cosmological constant along the wall, which means that the wall will have positive tension. Because gravity in $5 D$ will not be totally confined to the wall, and during inflation driven by a positive tension, the wall's gravitational field will repulse any objects in the bulk. This is a well known property of domain walls in flat $4 D$ spacetime [24], and all solutions with positive tension also share it. This could solve the bulk horizon problem as follows. When the expansion of the brane is fast, both the brane and bulk horizons are small, close to the fundamental scale. There will be many such sufficiently small causally isolated domains which are almost smooth both along the brane and around it. They can therefore start to inflate. Hence if inflation on the wall lasts long enough and proceeds slowly, the gravitational field of the wall will push out inhomogeneities in the bulk very far away from the wall. In this fashion, the bulk around the wall can also become homogeneous during wall inflation. Another interesting aspect is the interplay of positive bulk and brane cosmological terms, which can rapidly inflate only the wall, while leaving the bulk size almost constant. The resulting inflationary expansion is then highly asymmetric, of precisely the type discussed in the longwavelength limit in [12]. It may be necessary that in order to realize this possibility the bulk degrees of freedom must be very light, or very weakly coupled [6, 26, 10]. However, such models may emerge in theories which have large extra dimensions, and thus this is certainly a possibility [11, 27].

Writing down a concrete model is not as easy as it might seem, however. If the large internal dimensions are stabilized with the bulk size smaller than the horizon length on the wall, the approximation based on applying the usual $4 D$ scenarios and ignoring the gravitational effects in the extra dimensions is a good one, and scaled-down versions of models such as [28-31 may apply. If the extra dimensions are dynamical, it may happen that the $4 D$ scenarios are still applicable [12]. In other scenarios the effects of gravity in the extra dimensions may be significant, as we have seen here (see also [23]), and may require a different approach. An example we have noted here is the interplay of the wall and the bulk physics via gravity, manifest in the role of the bulk event horizon. This indicates that higher-dimensional dynamics in a generic situation warrants further investigation.

Note added in proof: While this paper was being submitted, we became aware of the work [35], which contains a subset of the solutions presented here.

\section{Acknowledgements}

We would like to thank S. Dimopoulos, E. Halyo, A. Linde and M. Luty for useful conversations. This work has been supported in part by NSF Grant PHY-9870115. 


\section{A Appendix}

We are looking for the solutions of the form (4). For the wall de-Sitter metric we chose the parametrization $d S_{d S}^{2}=\frac{-d \tau^{2}+d \vec{x}^{2}}{H^{2} \tau^{2}}$. These coordinates are related to familiar de-Sitter coordinates by $\tau=\kappa_{5}^{2 / 3} \exp (-H t), \vec{x}=\kappa_{5}^{2 / 3} H \vec{y}$. The brane curvature $H$ will be determined by the cosmological terms $\Lambda$ and $\sigma$, modulo a coordinate transformations. We will see that later. The $5 \mathrm{D}$ metric of interest is

$$
d s^{2}=a^{2}(w) \frac{-d \tau^{2}+d \vec{x}^{2}}{H^{2} \tau^{2}}+b^{2}(w) d w^{2}
$$

To get the reduction, we can evaluate the Ricci scalar for this metric. A straightforward calculation gives

$$
R=12 \frac{H^{2}}{a^{2}}-12 \frac{a^{\prime 2}}{a^{2} b^{2}}-\frac{8}{a b}\left(\frac{a^{\prime}}{b}\right)^{\prime}
$$

By our ansatz, $\sqrt{g}($ bulk $)=a^{4} b /(H \tau)^{4}$ and $\sqrt{g}$ (boundary $)=a^{4} /(H \tau)^{4}$, and so the volume of the de-Sitter space is $V_{4}=\int \frac{d \tau d^{3} \vec{x}}{H^{4} \tau^{4}}$. As is usual in supergravity models, we add to the action the usual Gibbons-Hawking term, which precisely cancels a total derivative term. Therefore the reduced effective action $I=2 \kappa_{5}^{2} S / V_{4}$ is

$$
I=\int d w\left\{12 H^{2} a^{2} b+12 \frac{a^{2} a^{\prime 2}}{b}+2 \kappa_{5}^{2} \Lambda b a^{4}\right\}-2 \kappa_{5}^{2} \int d w a^{4} \sigma \delta(w)
$$

where we have introduced the Dirac $\delta$ functions to represent the boundary terms by a fictitious bulk Lagrangian, in order to facilitate the variational procedure. To get the equations of motion, we now vary this action with respect to $b$ and $a$. After some simple algebra, with the gauge $b=1$, the equations become

$$
a^{\prime 2}=H^{2}+\frac{\kappa_{5}^{2} \Lambda}{6} a^{2} \quad a^{\prime \prime}-\frac{\kappa_{5}^{2} \Lambda}{6} a=-\frac{\kappa_{5}^{2}}{3} \sigma a(0) \delta(w)
$$

These equations are actually easy to solve. We can readily verify that the solution must be of the form

$$
a=\alpha \sinh \left(\sqrt{\frac{\kappa_{5}^{2} \Lambda}{6}}|w|\right)+\beta \cosh \left(\sqrt{\frac{\kappa_{5}^{2} \Lambda}{6}} w\right)
$$

to solve the second order differential equation. It is useful to recall that

$$
\begin{aligned}
\sinh \left(\sqrt{\frac{\kappa_{5}^{2} \Lambda}{6}}|w|\right) & =(2 \theta(w)-1) \sinh \left(\sqrt{\frac{\kappa_{5}^{2} \Lambda}{6}} w\right) \\
\left(\sinh \left(\sqrt{\frac{\kappa_{5}^{2} \Lambda}{6}}|w|\right)\right)^{\prime} & =\sqrt{\frac{\kappa_{5}^{2} \Lambda}{6}} \cosh \left(\sqrt{\frac{\kappa_{5}^{2} \Lambda}{6}} w\right)(2 \theta(w)-1) \\
\left(\sinh \left(\sqrt{\frac{\kappa_{5}^{2} \Lambda}{6}}|w|\right)\right)^{\prime \prime} & =\sqrt{\frac{2 \kappa_{5}^{2} \Lambda}{3}} \delta(w)+\frac{\kappa_{5}^{2} \Lambda}{6} \sinh \left(\sqrt{\frac{\kappa_{5}^{2} \Lambda}{6}}|w|\right)
\end{aligned}
$$


where $\theta(w)$ is the step function. Then using this, we find that $a^{\prime \prime}-\frac{\kappa_{5}^{2} \Lambda}{6} a=\sqrt{\frac{2 \kappa_{5}^{2} \Lambda}{3}} \alpha \delta(w)$ and hence the coefficients $\alpha$ and $\beta$ must satisfy

$$
\alpha=-\frac{\kappa_{5} \sigma}{\sqrt{6 \Lambda}} \beta
$$

This is the first relationship. The second relationship comes from the constraint equation: since $a^{\prime 2}-\frac{\kappa_{5}^{2} \Lambda}{6} a^{2}=H^{2}$, we get

$$
\alpha^{2}-\beta^{2}=\frac{6 H^{2}}{\kappa_{5}^{2} \Lambda}
$$

Using (46) and (47), we can evaluate $\alpha$ and $\beta$, and find

$$
\beta=\frac{6 H}{\kappa_{5} \sqrt{\kappa_{5}^{2} \sigma^{2}-6 \Lambda}} \quad \alpha=-\frac{\sqrt{6} \sigma H}{\sqrt{\Lambda} \sqrt{\kappa_{5}^{2} \sigma^{2}-6 \Lambda}}
$$

Therefore, the expression for the warp factor is

$$
a=\frac{6 H}{\kappa_{5} \sqrt{\kappa_{5}^{2} \sigma^{2}-6 \Lambda}}\left(\cosh \left(\sqrt{\frac{\kappa_{5}^{2} \Lambda}{6}} w\right)-\frac{\kappa_{5} \sigma}{\sqrt{6 \Lambda}} \sinh \left(\sqrt{\frac{\kappa_{5}^{2} \Lambda}{6}}|w|\right)\right)
$$

At the brane, $a(0)=\frac{6 H}{\kappa_{5} \sqrt{\kappa_{5}^{2} \sigma^{2}-6 \Lambda}}$, and we can normalize the coordinates along the brane such that their coefficient is unity, by the coordinate transformations $t \rightarrow \kappa_{5} \frac{\sqrt{\kappa_{5}^{2} \sigma^{2}-6 \Lambda}}{6 H} t$, and $\vec{x} \rightarrow \kappa_{5} \frac{\sqrt{\kappa_{5}^{2} \sigma^{2}-6 \Lambda}}{6 H} \vec{x}$, and the expansion rate along the brane $H=\kappa_{5} \sqrt{\kappa_{5}^{2} \sigma^{2}-6 \Lambda} / 6$ in comoving units. The normalized warp factor is

$$
a=\left(\cosh \left(\sqrt{\frac{\kappa_{5}^{2} \Lambda}{6}} w\right)-\frac{\kappa_{5} \sigma}{\sqrt{6 \Lambda}} \sinh \left(\sqrt{\frac{\kappa_{5}^{2} \Lambda}{6}}|w|\right)\right)
$$

When $\Lambda<0$ (positive cosmological constant), the hyperbolic functions in (54) should be replaced by trigonometric ones. In this case, the warp factor is, after normalizing it to unity on the brane with tension $\sigma$,

$$
a=\left(\cos \left(\sqrt{\frac{\kappa_{5}^{2}|\Lambda|}{6}} w\right)-\frac{\kappa_{5} \sigma}{\sqrt{6|\Lambda|}} \sin \left(\sqrt{\frac{\kappa_{5}^{2}|\Lambda|}{6}}|w|\right)\right)
$$

and the expansion rate is $H^{2}=\kappa_{5}^{2} \frac{\kappa_{5}^{2} \sigma^{2}+6|\Lambda|}{36}$. This completes our search for the single brane solutions.

Let us now consider parallel branes. We will look for the solution with two branes by taking (10) which describes one brane in $A d S_{5}$ as a background, and will fit the other brane in it, adjusting its tension as required. We will only consider the cases where the 
bulk geometry can be foliated by a congruence of slices which are conformal to the 3branes. Then away from the branes the original solution (44) would continue to solve the first of the eqs. (43), which explicitly gives

$$
a^{\prime 2}=\frac{\kappa_{5}^{4} \sigma^{2}}{36}-\frac{\kappa_{5}^{2} \Lambda}{6}+\frac{\kappa_{5}^{2} \Lambda}{6} a^{2}
$$

for $a$ given by (44) and with $\beta=1, \alpha=-\kappa_{5} \sigma / \sqrt{6 \Lambda}$ (which corresponds to the brane expansion rate $\left.H=\kappa_{5} \sqrt{\kappa_{5}^{2} \sigma^{2}-6 \Lambda} / 6\right)$. The second equation in this case is

$$
a^{\prime \prime}-\frac{\kappa_{5}^{2} \Lambda}{6} a=-\frac{\kappa_{5}^{2}}{3} \sigma a(0) \delta(w)-\frac{\kappa_{5}^{2}}{3} \bar{\sigma} a\left(w_{c}\right) \delta\left(w-w_{c}\right)
$$

It is evident that (44) will satisfy this equation away from the branes, where $\delta$-functions vanish. To take into account the effect of the branes, we compactify the bulk on the circle, $w \sim w+w_{c}$, and further orbifold the circle by $Z_{2}$ operation $w \sim-w$. Then we can substitute (44) into (53) and after some simple algebra find the required matching condition:

$$
\left(\sqrt{\frac{2 \kappa_{5}^{2} \Lambda}{3}}+\frac{\kappa_{5}^{3} \sigma \bar{\sigma}}{3 \sqrt{6 \Lambda}}\right) \sinh \left(\sqrt{\frac{\kappa_{5}^{2} \Lambda}{6}} w_{c}\right)=\frac{\kappa_{5}^{2}}{3}(\sigma+\bar{\sigma}) \cosh \left(\sqrt{\frac{\kappa_{5}^{2} \Lambda}{6}} w_{c}\right)
$$

The solutions of (54) are controlled by the signs of $\sigma, \bar{\sigma}$ and the ratios $\frac{\sqrt{6 \Lambda}}{\kappa|\sigma|}, \frac{\sqrt{6 \Lambda}}{\kappa|\bar{\sigma}|}$. Bearing in mind that $w_{c}>0$ (we ignore branes lying on top of each other since they are indistinguishable from single branes in our approach), and focusing first on $\Lambda>0$ case, it is straightforward to verify that solutions exist in the following cases:

- if $\sigma, \bar{\sigma}>0$ and $6 \Lambda>\max \left\{\kappa_{5}^{2} \sigma^{2}, \kappa_{5}^{2} \bar{\sigma}^{2}\right\}$ or $6 \Lambda<\min \left\{\kappa_{5}^{2} \sigma^{2}, \kappa_{5}^{2} \bar{\sigma}^{2}\right\}$. This solution is qualitatively different from examples considered by [15]. The distance between the branes is

$$
\tanh \left(\sqrt{\frac{\kappa_{5}^{2} \Lambda}{6}} w_{c}\right)=\frac{\kappa_{5} \sqrt{6 \Lambda}(\sigma+\bar{\sigma})}{6 \Lambda+\kappa_{5}^{2} \sigma \bar{\sigma}}
$$

while the warp factor on the other brane is

$$
a\left(w_{c}\right)=\sqrt{\left|\frac{6 \Lambda-\kappa_{5}^{2} \sigma^{2}}{6 \Lambda-\kappa_{5}^{2} \bar{\sigma}^{2}}\right|}
$$

In this case, the warp factor on the other brane can be both larger or smaller than unity, depending on the ratio of the tensions $\sigma / \bar{\sigma}$. If they are equal, then the warp factor on both branes is exactly unity, and the branes are at a distance $\tanh \left(\sqrt{\frac{\kappa_{5}^{2} \Lambda}{6}} w_{c}\right)=\frac{2 \sqrt{6 \Lambda} \kappa_{5} \sigma}{6 \Lambda+\kappa_{5} \sigma^{2}}$.

- either $\sqrt{\Lambda}=\frac{\kappa_{5} \sigma}{\sqrt{6}}$ or $\sqrt{\Lambda}=\frac{\kappa_{5} \bar{\sigma}}{\sqrt{6}}$; for both cases the constraint (54) reduces to $(\sigma+$ $\bar{\sigma}) \sinh \left(\sqrt{\frac{\kappa_{5}^{2} \Lambda}{6}} w_{c}\right)=(\sigma+\bar{\sigma}) \cosh \left(\sqrt{\frac{\kappa_{5}^{2} \Lambda}{6}} w_{c}\right)$. The only solutions of this equation are $\bar{\sigma}=-\sigma$, when the distance between the branes $w_{c}$ can be arbitrary, which is precisely the solution of [15], or $w_{c} \rightarrow \infty$ if $\sigma+\bar{\sigma} \neq 0$, which reduces to a single brane solution. 
- if $\sigma>0$ and $\bar{\sigma}<0$ but $\sigma+\bar{\sigma}<0$, there exist solutions when $6 \Lambda<\kappa_{5}^{2} \sigma^{2}$. The distance between the two branes is determined by the equation

$$
\tanh \left(\sqrt{\frac{\kappa_{5}^{2} \Lambda}{6}} w_{c}\right)=\frac{\kappa_{5} \sqrt{6 \Lambda}(|\bar{\sigma}|-\sigma)}{\kappa_{5}^{2} \sigma|\bar{\sigma}|-6 \Lambda}
$$

Note that the solution converges to that of [15] in the simultaneous limit $\sigma=|\bar{\sigma}|=\frac{\sqrt{6 \Lambda}}{\kappa_{5}}$. The warp factor on the brane with negative tension

$$
a\left(w_{c}\right)=\sqrt{\frac{\kappa_{5}^{2} \sigma^{2}-6 \Lambda}{\kappa_{5}^{2}|\bar{\sigma}|^{2}-6 \Lambda}}
$$

and is smaller than unity. In the limit $\sigma=|\bar{\sigma}|=\frac{\sqrt{6 \Lambda}}{\kappa_{5}}$ the ratio becomes undetermined. This is equivalent to the fact that the distance between the branes is arbitrary in this specific limit.

- if $\sigma>0, \bar{\sigma}<0$ but $\sigma+\bar{\sigma}>0$, the solutions exist if $6 \Lambda>\kappa_{5}^{2} \sigma^{2}$. In this case, the distance between the branes is

$$
\tanh \left(\sqrt{\frac{\kappa_{5}^{2} \Lambda}{6}} w_{c}\right)=\frac{\kappa_{5} \sqrt{6 \Lambda}(\sigma-|\bar{\sigma}|)}{6 \Lambda-\kappa_{5}^{2} \sigma|\bar{\sigma}|}
$$

while the warp factor at the location of the second brane is

$$
a\left(w_{c}\right)=\sqrt{\frac{6 \Lambda-\kappa_{5}^{2} \sigma^{2}}{6 \Lambda-\kappa_{5}^{2}|\bar{\sigma}|^{2}}}
$$

Note that in this case the warp factor at the negative tension brane is greater than unity. Once more, in the limit $\sigma=|\bar{\sigma}|=\frac{\sqrt{6 \Lambda}}{\kappa_{5}}$ both the warp factor and the distance between the branes become undetermined.

We now consider parallel branes when $\Lambda<0$. All of these solutions can be thought of as two parallel inflating branes in the orbifold construction. Again, by the orbifolding condition requres $w_{c}>0$. Then the matching condition (54) is replaced by

$$
\left(\frac{\kappa_{5}^{3} \sigma \bar{\sigma}}{3 \sqrt{6|\Lambda|}}-\sqrt{\frac{2 \kappa_{5}^{2}|\Lambda|}{3}}\right) \sin \left(\sqrt{\frac{\kappa_{5}^{2}|\Lambda|}{6}} w_{c}\right)=\frac{\kappa_{5}^{2}}{3}(\sigma+\bar{\sigma}) \cos \left(\sqrt{\frac{\kappa_{5}^{2}|\Lambda|}{6}} w_{c}\right)
$$

The solutions of this equation can be divided into three categories:

- if $\bar{\sigma}=-\sigma$, we must have $\sin \left(\sqrt{\frac{\kappa_{5}^{2}|\Lambda|}{6}} w_{c}\right)=0$, leading to the interbrane distance

$$
w_{c}=\sqrt{\frac{6}{\kappa_{5}^{2}|\Lambda|}} \pi
$$


In this case, the warp factor on the brane with the negative tension is $a=-1$. The sign change shows that there is a Rindler horizon between the branes. Its precise location is given by

$$
w_{H}=\sqrt{\frac{6}{\kappa_{5}^{2}|\Lambda|}} \tan ^{-1}\left(\frac{\kappa_{5} \sigma}{\sqrt{6|\Lambda|}}\right)
$$

The brane with the negative tension attracts every object in the bulk, whereas the brane with the positive tension repells them, although both are exponentially inflating.

- if $\sigma>0, \bar{\sigma}>0$ and $6|\Lambda|=\kappa_{5}^{2} \sigma \bar{\sigma}$, the solutions must satisfy $\cos \left(\sqrt{\frac{\kappa_{5}^{2}|\Lambda|}{6}} w_{c}\right)=0$,

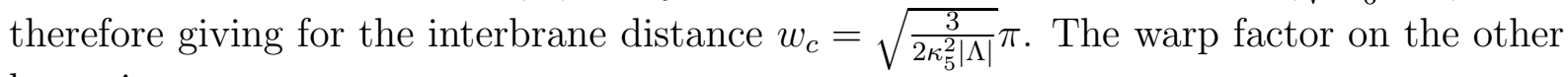
brane is

$$
a\left(w_{c}\right)=-\frac{\kappa_{5} \sigma}{\sqrt{6|\Lambda|}}
$$

Hence there is still a horizon between the branes, although both have positive tension. The reason for this is that the brane with larger tension is more repulsive than the one with smaller tension.

- if $\sigma+\bar{\sigma} \neq 0$ and $6|\Lambda| \neq \kappa_{5}^{2} \sigma \bar{\sigma}$, the solutions must satisfy

$$
\tan \left(\sqrt{\frac{\kappa_{5}^{2}|\Lambda|}{6}} w_{c}\right)=\frac{\kappa_{5} \sqrt{6|\Lambda|}(\sigma+\bar{\sigma})}{\kappa_{5}^{2} \sigma \bar{\sigma}-6|\Lambda|}
$$

This equation determines the separation between the branes. It is given by

$$
w_{c}=\sqrt{\frac{6}{\kappa_{5}^{2}|\Lambda|}}\left(\tan ^{-1}\left(\frac{\kappa_{5} \sqrt{6|\Lambda|}(\sigma+\bar{\sigma})}{\kappa_{5}^{2} \sigma \bar{\sigma}-6|\Lambda|}\right)+2 n \pi\right)
$$

The warp factor is then given by

$$
a\left(w_{c}\right)=\zeta \sqrt{\frac{6|\Lambda|+\kappa_{5}^{2} \sigma^{2}}{6|\Lambda|+\kappa_{5}^{2} \bar{\sigma}^{2}}}
$$

where $\zeta=-\operatorname{sgn}\left\{\cos \left(\sqrt{\frac{\kappa_{5}^{2}|\Lambda|}{6}} w_{c}\right)\right\}$. Thus, there may or may not be a horizon between the branes, depending on the interplay of the three terms $\sigma, \bar{\sigma}$ and $|\Lambda|$.

\section{References}

[1] N. Arkani-Hamed, S. Dimopoulos and G. Dvali, Phys. Lett. B429, 263 (1998); hepph/9807344; I. Antoniadis, N. Arkani-Hamed, S. Dimopoulos and G. Dvali, Phys. Lett. B436, 257 (1998). 
[2] I. Antoniadis, Phys. Lett. B246, 377 (1990); I. Antoniadis and K. Benakli, Phys. Lett. B326 69 (1994); I. Antoniadis, K. Benakli and M. Quiros, Phys. Lett. B331 313 (1994).

[3] N. Arkani-Hamed, S. Dimopoulos and J. March-Russell, hep-th/9809124.

[4] K. Dienes, E. Dudas and T. Gherghetta, Phys. Lett. B436, 55 (1998); hepph/9806292; hep-ph/9807522; K. Dienes, E. Dudas, T. Gherghetta and A. Riotto, hep-ph/9809406.

[5] R. Sundrum, hep-ph/9805471; hep-ph/9807348; G. Shiu and S.-H. Tye, Phys. Rev. D58, 106007 (1998); Z. Kakushadze and S.-H. Tye, hep-th/9809147; C. Bachas, hep-ph/9807415; K. Benakli, eprint hep-ph/9809582; L. Randall and R. Sundrum, hep-th/9810155.

[6] K. Benakli and S. Davidson, hep-ph/9810280.

[7] P. Hořava and E. Witten, Nucl. Phys. B460, 506 (1996); E. Witten, Nucl. Phys. 471, 135 (1996).

[8] J.D. Lykken, Phys. Rev. D54, 3693 (1996); I. Antoniadis and M. Quiros, Phys. Lett. B392, 61 (1997); C.P. Burgess, L.E. Ibanez and F. Quevedo, hep-ph/9810535; L.E. Ibanez, C. Munoz and S. Rigolin, hep-ph/9812397; I. Antoniadis, G. D'Appollonio, E. Dudas and A. Sagnotti, hep-th/9812118.

[9] G.F. Giudice, R. Rattazzi and J.D. Wells, hep-ph/9811291; M. Maggiore and A. Riotto, hep-th/9811089; S. Nussinov and R. Shrock, hep-ph/9811323; E.A. Mirabelli, M. Perelstein and M.E. Peskin, hep-ph/9811337; T. Han, J.D. Lykken and R. Zhang, hep-ph/9811350; J.L. Hewett, hep-ph/9811356; Z. Berezhiani and G. Dvali, hep-ph/9811378; K.R. Dienes, E. Dudas and T. Gherghetta, hep-ph/9811428; N. Arkani-Hamed, S. Dimopoulos, G. Dvali and J. March-Russell, hep-ph/9811448; Z. Kakushadze, hep-th/9811193; hep-th/9812163; I. Antoniadis and C. Bachas, hepth/9812093; S. Cullen and M. Perelstein, hep-ph/9903422.

[10] N. Kaloper and A. Linde, hep-th/9811141.

[11] G. Dvali and S.H.H. Tye, hep-ph/9812483.

[12] N. Arkani-Hamed, S. Dimopoulos, N. Kaloper and J. March-Russell, hep-ph/9903224; hep-ph/9903239.

[13] V. Rubakov and M. Shaposhnikov, Phys. Lett. B125, 136 (1983); A.T. Barnaveli and O.V. Kancheli, Sov. J. Nucl. Phys. 52, 576 (1990).

[14] G. Dvali and M. Shifman, Nucl. Phys. B504, 127 (1997).

[15] L. Randall and R. Sundrum, hep-ph/9905221. 
[16] P.G.O. Freund and M.A. Rubin, Phys. Lett. B97 233 (1980); F. Englert, Phys. Lett. B119 339 (1982); M.J. Duff, B.E.W. Nilsson and C.N. Pope, Phys. Rev. Lett. 502043 (1983).

[17] J. Maldacena, Adv. Theor. Math. Phys. 2231 (1998); P. Claus, R. Kallosh, J. Kumar, P. Townsend and A. Van Proeyen, JHEP 9806004 (1998).

[18] H. Lu, C.N. Pope and P.K. Townsend, Phys. Lett. B391 39 (1997); P.M. Cowdall, H. Lu, C.N. Pope, K.S. Stelle and P.K. Townsend, Nucl. Phys. B486 49 (1997); M.S. Bremer, M.J. Duff, H. Lu, C.N. Pope and K.S. Stelle, Nucl. Phys. B543 321 (1999); M. Cvetic, J.T. Liu, H. Lu and C.N. Pope, hep-th/9905096.

[19] M. Cvetic, S. Griffies and H. Soleng, Phys. Rev. D48 2613 (1993); M. Cvetic and H. Soleng, Phys. Rept. 282159 (1997).

[20] A. Lukas, B.A. Ovrut, K.S. Stelle and D. Waldram, Phys. Rev. D59, 086001 (1999).

[21] A. Lukas, B.A. Ovrut and D. Waldram, hep-th/9902071.

[22] S.W. Hawking and G.F.R. Ellis, The large scale structure of space-time, Cambridge University Press, Cambridge 1973.

[23] P. Binetruy, C. Deffayet and D. Langlois, hep-th/9905012.

[24] A. Vilenkin, Phys. Lett. 133177 (1983); J. Ipser and P. Sikivie, Phys. Rev. D30 712 (1984).

[25] G.W. Gibbons and S.W. Hawking, Phys. Rev. D15, 2738 (1977); S.W. Hawking and I.G. Moss, Phys. Lett. B110, 35 (1982); W. Boucher and G.W. Gibbons, in The Very Early Universe, ed. G.W. Gibbons, S.W. Hawking and S. Siklos, Cambridge University Press, Cambridge (1983); A.A. Starobinsky, JETP Lett. 37, 66 (1983); R. Wald, Phys. Rev. D28, 2118 (1982).

[26] D. Lyth, hep-ph/9810320.

[27] E. Halyo, hep-ph/9904432; hep-ph/9905244.

[28] A. Linde, Phys. Lett. B129, 177 (1983).

[29] A. Linde, Phys. Lett. B259, 38 (1991); Phys. Rev. D49, 748 (1994);

[30] E.J. Copeland, A.R. Liddle, D.H. Lyth, E.D. Stewart and D. Wands, Phys. Rev. D49, 6410 (1994); E.D. Stewart, Phys. Rev. D51, 6847 (1995).

[31] P. Binetruy and G. Dvali, Phys. Lett. B388, 241 (1996); E. Halyo, Phys. Lett. B387, 43 (1996); A.D. Linde and A. Riotto, Phys. Rev. D56, 1841 (1997).

[32] T. Banks, M. Dine and A. Nelson, hep-th/9903019. 
[33] H.A. Chamblin and H.S. Reall, hep-th/9903225; P. Kanti and K.A. Olive, hepph/9903524; J.M. Cline, hep-ph/9904495; A. Riotto, hep-ph/9904485.

[34] G. Dvali and G. Gabadadze, hep-ph/9904221; G. Dvali and M. Shifman, hepth/9904021; G. Dvali, hep-ph/9905204.

[35] T. Nihei, hep-ph/9905487. 